\begin{tabular}{|c|c|}
\hline Title & High-performance absorbing boundary conditions for photonic crystal waveguide simulations \\
\hline Author(s) & Koshiba, Masanori; Tsuji, Y asuhide; Sasaki, Satoru \\
\hline Citation & $\begin{array}{l}\text { Microwave and Wireless Components Letters, 11(4), 152-154 } \\
\text { https://doi.org/10.1109/260.916327 }\end{array}$ \\
\hline Issue Date & 2001-04 \\
\hline Doc URL & http:/hdl. handle.net/2115/5583 \\
\hline Rights & $\begin{array}{l}\text { O2001 IEEE. Personal use of this material is permitted. However, permission to reprint/republish this material for } \\
\text { advertising or promotional purposes or for creating new collective works for resale or redistribution to servers or lists, } \\
\text { or to reuse any copyrighted component of this work in other works must be obtained from the IEEE." }\end{array}$ \\
\hline Type & article \\
\hline File Information & IMWCL11-4.pdf \\
\hline
\end{tabular}

Instructions for use 


\title{
High-Performance Absorbing Boundary Conditions for Photonic Crystal Waveguide Simulations
}

\author{
Masanori Koshiba, Senior Member, IEEE, Yasuhide Tsuji, Member, IEEE, and Satoru Sasaki
}

\begin{abstract}
A high-performance absorbing boundary condition is newly developed for the reduction of spurious reflections in photonic crystal (PC) waveguide simulations. The $P C$ waveguide is terminated with a perfectly matched layer (PML) in which the original PC structure remains as is. This PC-based PML works well, compared to a conventional PML, which acts as a homogeneous absorbing medium, simulating a semi-infinite free space and to a distributed-Bragg-reflector waveguide, which was recently developed to reduce reflections from PC waveguide ends, improving a wavenumber matching condition for $\mathrm{PC}$ waveguide modes.
\end{abstract}

Index Terms-Absorbing boundary condition, perfectly matched layer, photonic crystal, photonic crystal waveguide, time-domain analysis.

\section{INTRODUCTION}

$\mathbf{P}$ HOTONIC crystals (PCs) have inspired great interest because of their potential ability to control the lightwave propagation. Mekis et al. [1] have demonstrated high transmission through a sharp $90^{\circ}$ bend in PC waveguides by using the finite-difference time-domain (FDTD) method. When simulating PC waveguide devices and/or circuits, in order to reduce spurious reflections from the computational window edges, the use of appropriate absorbing boundary conditions is indispensable. For this purpose, the so-called perfectly matched layer (PML) condition [2] has been applied to PC waveguide simulations [1]. Although the impedance matching condition is satisfied at the interface between the PML region and the non-PML, real PC region, substantial reflection was observed [1], [3]. Recently, Mekis et al. [3] have proposed to terminate a PC waveguide with a distributed-Bragg-reflector (DBR) waveguide improving the wavenumber matching condition for PC waveguide modes. However, this condition can be exactly satisfied at only one frequency, and the reflection level was still on the order of a few percent. In this paper a high-performance absorbing boundary condition for PC waveguide simulations will be presented.

We consider a two-dimensional PC waveguide $(\partial / \partial x=0)$ surrounded by PMLs of thickness $d_{i}(i=1$ to 4$)$ as shown in Fig. 1, in which the original PC structure remains as is, hereafter, referred to as PC-based PML. Using the anisotropic PML [4],

Manuscript received October 2, 2000; revised January 24, 2001. This work was supported in part by a Grant-in-Aid for Scientific Research from the Ministry of Education, Science, and Culture, Japan, and by CREST, Japan Science and Technology Corporation (JST), Japan. The review of this letter was arranged by Associate Editor Dr. Arvind Sharma.

The authors are with the Division of Electronics and Information Engineering, Hokkaido University, Sapporo 060-8628, Japan.

Publisher Item Identifier S 1531-1309(01)03330-X

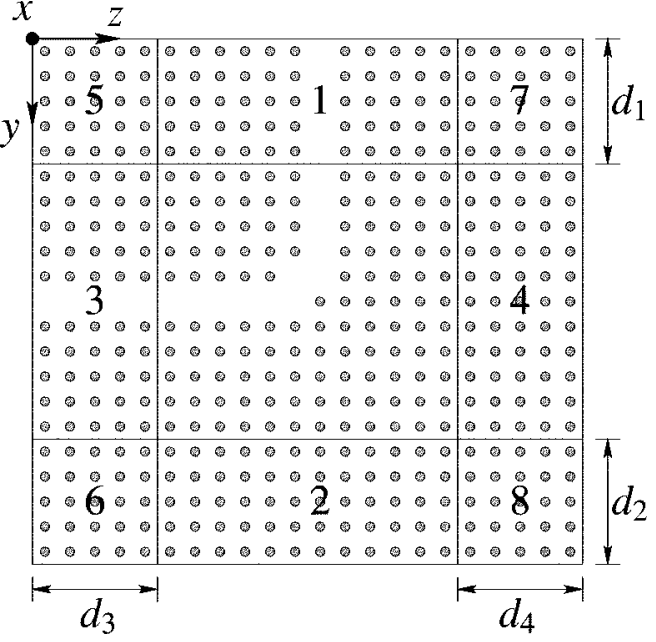

Fig. 1. Photonic crystal (PC) waveguide surrounded by PC-based perfectly matched layer (PML) regions 1 to 8.

[5], the PML permittivity and permeability tensors are written as

$$
[\varepsilon]_{\mathrm{PML}}=\varepsilon_{0} n^{2}[\Lambda], \quad[\mu]_{\mathrm{PML}}=\mu_{0}[\Lambda]
$$

with

$$
[\Lambda]=\left[\begin{array}{ccc}
s_{y} s_{z} / s_{x} & 0 & 0 \\
0 & s_{z} s_{x} / s_{y} & 0 \\
0 & 0 & s_{x} s_{y} / s_{z}
\end{array}\right]
$$

where $\varepsilon_{0}$ and $\mu_{0}$ are permittivity and permeability of free space, $n$ is the refractive index, and the PML parameters, $s_{x}, s_{y}$, and $s_{z}$, are given in Table I. Assuming parabolic profiles of artificial electric and magnetic conductivities, $\sigma$ and $\sigma^{*}$, the value of $s_{i}$ in Table I is taken as

$$
s_{i}=1-j\left(\rho / d_{i}\right)^{2} \tan \delta_{i}
$$

with

$$
\tan \delta_{i}=\frac{\sigma_{i, \max }}{\omega \varepsilon_{0} n_{i 0}^{2}}=\frac{\sigma_{i, \max }^{*}}{\omega \mu_{0}}
$$

where $\rho$ is the distance from the beginning of PML (PML surface), $\delta_{i}$ is the loss angle at the end of PML $\left(\rho=d_{i}\right), \omega$ is the angular frequency, $n_{i 0}$ is the refractive index at the PML surface $(\rho=0)$, subscript "max" is the maximum value, and (4) is required to satisfy the impedance matching condition at 
TABLE I

PML PARAMETERS

\begin{tabular}{ccccccccc}
\hline \multirow{8}{*}{$\begin{array}{c}\text { PML } \\
\text { parameter }\end{array}$} & 1 & 2 & 3 & 4 & 5 & 6 & 7 & 8 \\
\hline$s_{y}$ & $s_{1}$ & $s_{2}$ & 1 & 1 & $s_{1}$ & $s_{2}$ & $s_{1}$ & $s_{2}$ \\
$s_{z}$ & 1 & 1 & $s_{3}$ & $s_{4}$ & $s_{3}$ & $s_{3}$ & $s_{4}$ & $s_{4}$ \\
\hline$s_{x}=1$ in all the PML regions. & & & &
\end{tabular}

TABLE II

DEFINITION OF $\Phi, p$, AND $q$

\begin{tabular}{cccc}
\hline mode & $\Phi$ & $p$ & $q$ \\
\hline TE & $E_{x}$ & 1 & $n^{2}$ \\
TM & $H_{x}$ & $1 / n^{2}$ & 1 \\
\hline
\end{tabular}

the interface between the PML region and the real PC region $\left(s_{x}=s_{y}=s_{z}=1\right)$.

From Maxwell's equations with (1) and (2), we obtain

$$
\frac{\partial}{\partial y}\left(p \frac{s_{z}}{s_{x} s_{y}} \frac{\partial \Phi}{\partial y}\right)+\frac{\partial}{\partial z}\left(p \frac{s_{y}}{s_{x} s_{z}} \frac{\partial \Phi}{\partial z}\right)-\frac{s_{y} s_{z}}{s_{x}} \frac{q}{c^{2}} \frac{\partial^{2} \Phi}{\partial t^{2}}=0
$$

where $c$ is the light velocity in free space, and $\Phi, p, q$ are defined in Table II with $E_{x}$ and $H_{x}$ being, respectively, the $x$ components of the electric and magnetic fields. To solve (5) in time domain, we adopt a recently developed time-domain beam propagation method based on a finite element scheme [6] which can treat a slowly varying envelope of a pulsed wave. In the time-domain analysis, $\omega$ in (4) is regarded as a carrier center angular frequency, $\omega_{c}$.

In order to quantify reflection properties when a PC-based PML is applied to a PC waveguide, we carry out numerical simulations of pulse propagation in a PC straight waveguide [3]. The waveguide is terminated with a conventional PML, a distributed-Bragg-reflector (DBR) waveguide [3], and a PC-based PML as shown, respectively, in Fig. 2(a)-(c), where dielectric rods with circular cross-section of radius $r=0.2 a$ and refractive index $n=3.4$ are embedded in air on a square array with lattice constant $a$. The waveguide, which runs along the middle of the cell, at the distance of $6 a$ from the edges, is designed long enough that at the cell center there is virtually no overlap between the original pulse and the pulse reflected from the waveguide end. This PC has a photonic band gap (PBG) for TE modes which extends from $\omega a /(2 \pi c)=0.283$ to 0.424 , but not for $\mathrm{TM}$ modes. Due to the exponential decay of guided modes within PBG, the fields are very small at the cell boundaries parallel to the waveguide and thus, special care is not necessary at these boundaries.

The measured reflection coefficient is shown as a function of normalized angular frequency in Fig. 3, where the inputted TE Gaussian pulse has a carrier center angular frequency $\omega_{c} a /(2 \pi c)=0.368$, and the reflection amplitudes from the PC waveguide end terminated with a conventional PML, a DBR waveguide, and a PC-based PML are, respectively, calculated from FDTD [3] (also checked by using FETD-BPM), FDTD [3], and FETD-BPM.

When using a conventional PML, substantial reflection is observed on the order of 20 to $30 \%$. When using a DBR waveguide,

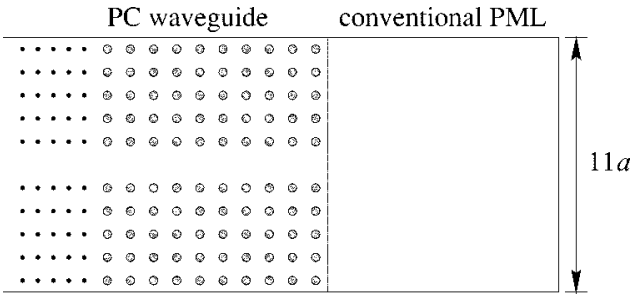

(a)

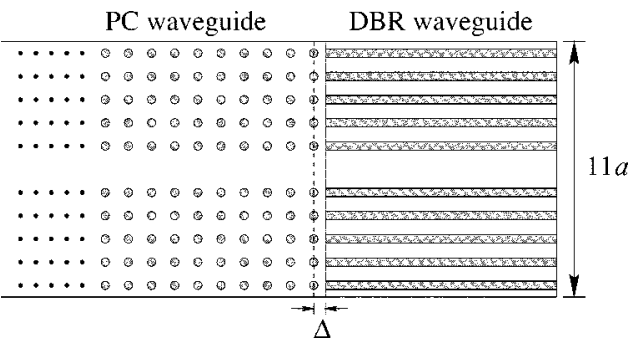

(b)

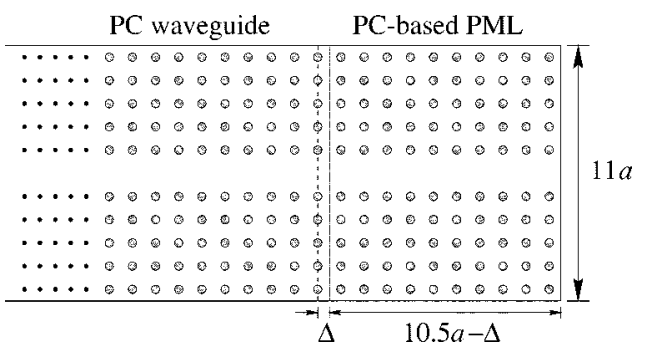

(c)

Fig. 2. PC straight waveguides terminated with (a) conventional PML, (b) DBR waveguide, and (c) PC-based PML.

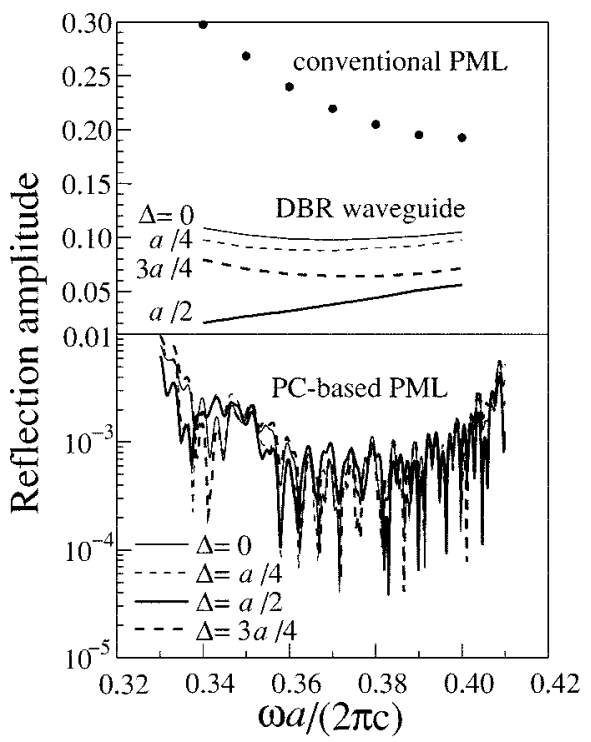

Fig. 3. Reflection amplitudes for conventional PML, DBR waveguide, and PC-based PML terminations.

there is a large improvement over the reflection from the conventional PML termination, but it is still on the order of a few percent. Also, the reflection level depends on the distance $\Delta$ between the beginning of DBR waveguide and the line defined by the centers of the last row of rods within the real PC region, and the lowest reflection is obtained for $\Delta=a / 2$. When using a PC-based PML proposed here, on the other hand, the reflection 


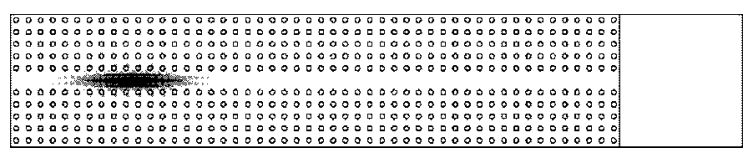

(a)

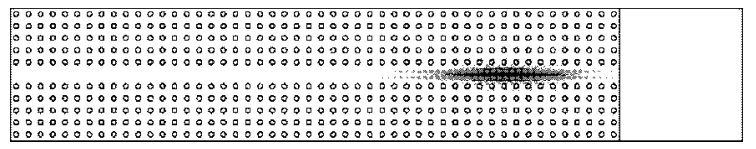

(b)

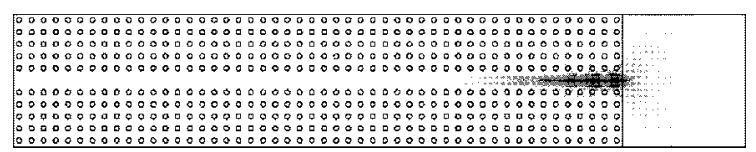

(c)

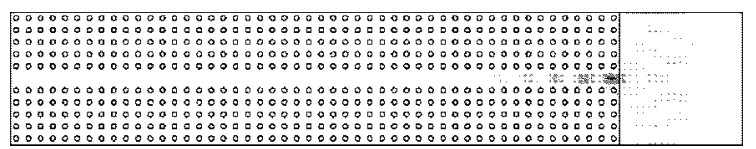

(d)

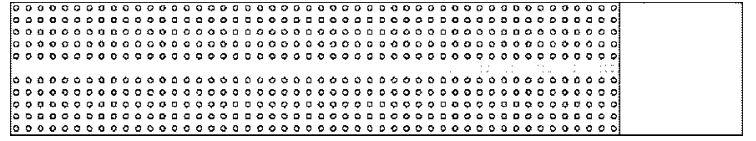

(e)

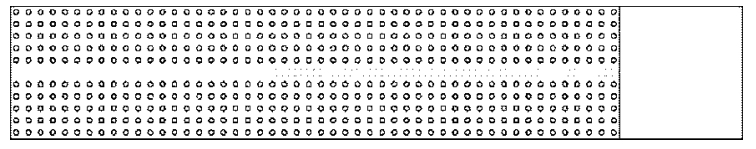

(f)

Fig. 4. Electric field patterns for conventional PML termination. (a) $t=0 \mathrm{fs}$ (incident pulse). (b) $t=120 \mathrm{fs}$. (c) $t=150$ fs. (d) $t=180 \mathrm{fs}$. (e) $t=210 \mathrm{fs}$. (f) $t=240 \mathrm{fs}$.

amplitude is drastically reduced and is less than $-50 \mathrm{~dB}$ across a wide range of frequencies, irrespective of values of $\Delta$. It should be noted that the reflection is larger at low and high frequencies near PBG edges.

Electric field patterns for the conventional PML and the PC-based PML terminations are, respectively, shown in Figs. 4 and 5. When using a conventional PML, we can see substantial reflection from the PML surface. When using a PC-based PML, on the other hand, the reflected field can be handly observed. A PC-based PML works well, compared to a conventional PML, because pulsed waves experience the same permittivity contrast as in the real PC region. A conventional PML, on the other hand, has not such a permittivity contrast and thus, acts as a homogeneous absorbing medium. The present PC-based PML is effective not only for guided modes confined in defect regions but for nonguided, radiated fields.

In conclusion, we have proposed a high-performance PC-based PML for PC waveguide simulations. This provides a simple means for reducing spurious reflections from computa-

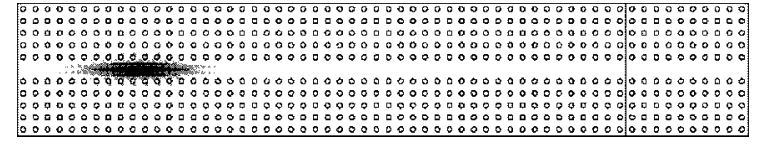

(a)

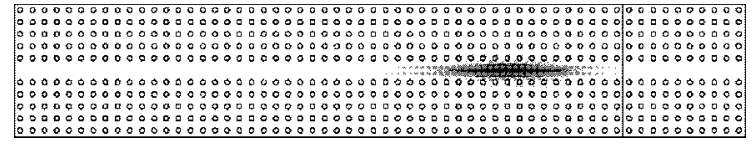

(b)

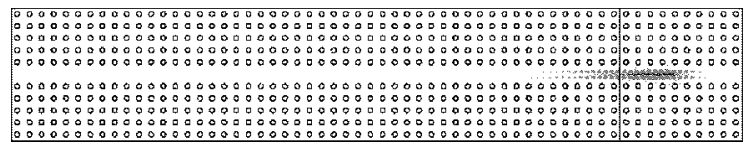

(c)

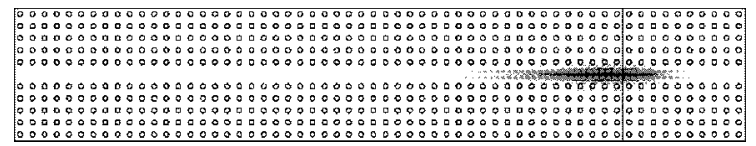

(d)

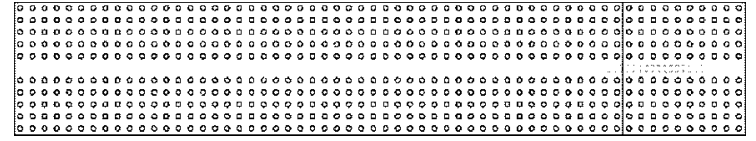

e)

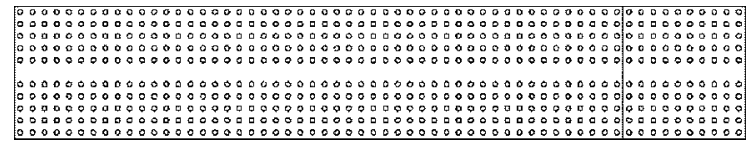

(f)

Fig. 5. Electric field patterns for PC-based PML termination. (a) $t=0 \mathrm{fs}$ (incident pulse). (b) $t=120$ fs. (c) $t=150$ fs. (d) $t=180$ fs. (e) $t=210$ fs. (f) $t=240 \mathrm{fs}$

tional window edges, compared to the conventional PML and the PBG waveguide terminations.

\section{REFERENCES}

[1] A. Mekis, J. C. Chen, I. Kurland, S. Fan, P. R. Villeneuve, and J. D. Joannopoulos, "High transmission through sharp bends in photonic crystal waveguides," Phys. Rev. Lett., vol. 77, pp. 3787-3790, Oct. 1996.

[2] J.-P. Berenger, "A perfectly matched layer for the absorption of electromagnetic waves," J. Comput. Phys., vol. 114, pp. 185-200, Oct. 1994.

[3] A. Mekis, S. Fan, and J. D. Joannopoulos, "Absorbing boundary conditions for FDTD simulations of photonic crystal waveguides," IEEE Microwave Guided Wave Lett., vol. 9, pp. 502-504, Dec. 1999.

[4] J.-Y. Wu, D. M. Kingsland, J.-F. Lee, and R. Lee, "A comparison of anisotropic PML to Berenger's PML and its application to the finiteelement method for EM scattering," IEEE Trans. Antennas Propagat., vol. 45, pp. 40-50, Jan. 1999.

[5] F. L. Teixeira and W. C. Chew, "General closed-form PML constitutive linear media," IEEE Microwave Guided Wave Lett., vol. 8, pp. 223-225, June 1998.

[6] M. Koshiba, Y. Tsuji, and M. Hikari, "Time-domain beam propagation method and its application to photonic crystal circuits," J. Lightwave Technol., vol. 18, pp. 102-110, Jan. 2000. 